\title{
The relative merits of altered fractionation in lung cancer with some emphasis in small cell lung cancer
}

\author{
Andrew T. Turrisi, III \\ Department of Radiution Oncology, The University of Michigan Medical Center, Ann Arbor, Michigan, USA
}

\section{Introduction}

Although some have negated the progress that has been achieved in the last decade, there has been steady improvement in the median survivals and two-year survivals as compared to trials published and presented from the Ashford Castle, Ireland Meeting in 1983 [1]. In that year, the median survivals approximated one year; 2-year survivals were at best 14 to 17 per cent. There are a number of explanations for this including stage migration and selection factors, all of which influence patient entry onto trials. However, better chemotherapeutics with platinum etoposide-based programs (instead of cyclophosphamide doxorubicin-based programs) and improved abilities to deliver radiotherapy in concurrent or alternating schemes may have contributed to these improved results.

A major reason to add radiotherapy to combined modality trials in small cell lung cancer is the approximately $80 \%$ local failure rate with chemotherapy alone. Even with the addition of radiotherapy in low doses, the failure rate still approximates $80 \%$ and in higher doses it remains at roughly $30 \%$ [2]. The best local control rate published is $97 \%$ after 60 Gy was used with an infrequently used chemotherapy regimen [3]. The median and 2-year survival were too short, perhaps reflecting poor systemic control with this chemotherapy program.

\section{Methods}

Table 1 displays the factors that may influence the relative merits of different schedules and strategies in administration of thoracic radiotherapy. Dose must be discussed both in terms of physical dose, expressed in rad or Gy, and relative biologic dose, expressed as "RET's" or Gy-10's for acute effects and "NRET's" or Gy-3's for late effects. The relative

Correspondence to: A.T. Turrisi, 11I, M.D., Dept. of Radiation Oncology, The University of Michigan Medical Center, Ann Arbor, MI 48109, USA. 
biologic dose is influenced by the fractionation scheme, the time, the dose-per-fraction, the program of chemotherapy, and the factors listed in Table 1. Some chemotherapeutic agents may cause an increase in adverse effects. The aim of combined modality therapy is to provide synergistic or at least additive anti-tumor effects. When certain strategies of thoracic radiotherapy are employed and with particular chemotherapeutics, such as doxorubicin, bleomycin, mitomycin- $\mathrm{C}$, or nitrosoureas, the net effect may produce an increase in lung tissue toxicity. The strategy of the successful combined modality trial is to find ways to provide increased efficacy against the cancer without causing excess toxicity to normal tissues.

The volume is another important factor that needs attention in lung cancer trials. Although volumes have increased in lung cancer therapy, no critical analysis shows that larger volumes improve survival or significantly enhance local control. Many have increased the volumes because of the anatomic knowledge of the lymphatics, or their frequency of involvement or potential for failure in those sites. Because of the therapeutic successes achieved in lymphomas, such as Hodgkin's disease, which were expanded field radiotherapies, the analogy dictates to lung cancer radiotherapy portals.

The Looney Hypothesis integrated radiotherapy with chemotherapy in novel ways [4]. In the laboratory, this resulted in enhanced cure rates. When brought to the clinic, another objective sought was reduction of toxicity. Alternating weeks of chemotherapy with weeks of radiotherapy allow relatively full doses but commonly requires split-course radiotherapy. This strategy has been attempted in small cell lung cancer; however, with the chemotherapeutics used and in the schedules employed, despite increasing the dose from $\mathbf{4 5}$ to 55 , and then $65 \mathrm{~Gy}, 3$-year actuarial figures continued to show $70 \%$ control, no improvement in terms of local control [5]. Survival was adequate and comparable to other studies of the era, but the French studies that attempted alternating therapy failed to provide the desired goal of better local therapy with lesser toxicity [5]. One reason for local failure may be inadequate or suboptimal coverage of the tumor bed. Many thoracic radiotherapy programs have used posterior spinal cord blocks. In this era, with the use of simulation and CT-directed treatment planning systems, both inclusion of target volumes and exclusion of critical normal tissues can be guaranteed. Over the next decade we will need to focus on normal tissue complication. The spinal cord has been the focus of concern in the past. Today computerized scans aid in diagnosis and therapy planning. These systems facilitate radiation of the target, but also assure protection of critical tissues. Systems are now being tested to determine new levels for partial-organ tolerances. Prediction of complication may

TABLE 1

Factors which may influence the relative merits of thoracic radiotherapy

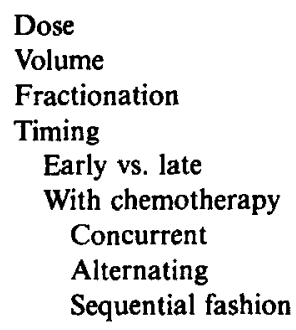


vary amongst different normal tissues that may have varied dependencies on volume, dose, and slope of curves describing specific events. For some tissues, like lung, volume is likely to be quite important, perhaps much more important than dose.

The concept of iso-effects is not new. When different fraction schemes are used, it is valuable to be able to compare the relative acute and late effects of various fraction schemes. The concepts of nominal standard dose (NSD) and biologic-effective dose (BED) draw attention to the fact that the physical dose by itself is an inadequate representation of the effect that radiotherapy has on living tissues [6]. Radiation oncologists have understood that there is a difference between the acute tissues' response and late effects, but most regimens have been designed to balance acute-effect tissue response. The correlation between acute effects and late effects is imperfect. However, acute effect may parallel antitumor effect. In the ideal world, it would be wonderful to have a therapy that produces no acute tissue damage, but it may be unrealistic to expect that there will be an absence of acute effects that still maintain anti-tumor effects. Today, we are better able to support patients through acutely toxic events.

Standard or conventional dose radiotherapy has presently been established at $60 \mathrm{~Gy}$ in 2-Gy fractions. However, precise control rates in the chest and the exact dose limiting toxicity to each of the organs irradiated remain somewhat arbitrarily defined, so many are unsatisfied with that, and we plan to attempt to re-establish partial lung tolerances for radiotherapy in standard fraction schemes by using 3-D treatment planning. However, standard fractionation already lasts for 6 weeks. The prospect of accelerated repopulation of cancer cells during these protracted schedules has led many to explore accelerated fraction schemes. Hyperfractionation is very good for tumors with no radiobiologic shoulders. Accelerated fraction schemes have been used to treat small cell lung cancer; however, some may want to test a hyperfractionated scheme, particularly if the late effects to lung and spinal cord of the accelerated schedules seem excessive [7]. Furthermore, most view the late cffects that have been achieved with these accelerated schedules to be fairly welltolerated. Table 2 compares the advantages of accelerated versus hyperfractionated schemes. In general, there has been a movement away from the use of hypofractionation, except for palliation.

\section{Clinical example}

In 1984, at the University of Pennsylvania, we conducted a trial comparing twice-a-day

TABLE 2

Advantages of hyperfractionation and acceleration

\begin{tabular}{ll}
\hline Hyperfractionation & Accelerated fractions \\
\hline $\begin{array}{l}\text { Excellent for sensitive tumors without shoulders } \\
\text { Respects delicate surrounding late normal tissues (i.e. } \\
\text { lung, spinal cord) }\end{array}$ & $\begin{array}{l}\text { Sensitivity relatively less important } \\
\text { Attends to accelerated "repopulations" }\end{array}$ \\
$\begin{array}{l}\text { Higher physical dose } \\
\text { Time relatively unchanged from standard therapy }\end{array}$ & $\begin{array}{l}\text { Lower physical dose increased biologic dose? } \\
\text { Decreased overall time } \\
\text { Relatively increased risk of acute toxicity }\end{array}$ \\
\hline
\end{tabular}


radiotherapy used concurrently with platinum etoposide chemotherapy $\left(60 \mathrm{mg} / \mathrm{m}^{2}\right.$ on day 1 , etoposide $120 \mathrm{mg} / \mathrm{m}^{2}$ days 4,6 , and 8 of each cycle); two intense cycles of that chemotherapy were used; the radiotherapy overlapped with the first cycle [7]. There were no weekend treatments. Breaks were not allowed in radiotherapy, and, irrespective of the Cycle 1 toxicity, Cycle 2 chemotherapy was given in full doses on day 22. Subsequently, six alternating cycles of platinum etoposide (PE) and cyclophosphamide, doxorubicin, vincristine were alternated. At completion of approximately 6 months of therapy, prophylactic cranial irradiation was applied to all surviving, completely responding patients. The rationale for using the twice-daily radiotherapy was that the small fractions cause relatively less damage to surrounding normal tissues with a radiobiologic shoulder, such as lung and spinal cord, and even relatively acute-responding tissues, such as esophagus. On the other hand, small cell lung cancers without a shoulder were exponentially killed; and thus, did not have the opportunity to repair the damage that these relatively small doses per fraction provided. Also, since small cell lung cancer has the most prolific growth rate of any solid tumor, the potential for tumor growth between fractions of radiotherapy made allowed for passage from the relatively resistant S-Phase of the cell cycle to the more sensitive G2-M or M-Phase which is more radiosensitive [7].

Table 3 displays the PE trials: five twice-a-day radiotherapy studies and two once-a-day radiotherapy studies. There appear to be improved outcomes between these trials and older cyclophosphamide-based trials. The two-year survival is not dramatic between the daily and b.i.d. schedules. A number of factors differ between the trials including: PE-dose schedule, timing of radiotherapy, selection criteria, duration of follow-up. The data presented show some differences in local control. However, the majority of failures remain systemic failures that lead to the largest cause of patient death. In order to clarify some of these issues, presently, there is an active ongoing trial in the ECOG, RTOG and SWOG that compares 45 Gy given in 3 weeks by a b.i.d. accelerated schedule versus 45 Gy given in 5 weeks in a standard schedule employing 1.8-Gy daily fractions. Early endpoints for toxicity, response and median survival appear similar; however, the longer-term endpoints of $1,2,3$ and 5 year disease free and overall survival, as well as local control, need more

TABLE 3

Combined thoracic radiotherapy and platinum etoposide trials for limited small cell lung cancer

\begin{tabular}{|c|c|c|c|c|c|}
\hline & No. of patients & Dose (Gy) & Time (weeks) & $\begin{array}{l}\text { Percentage 2- } \\
\text { year survival }\end{array}$ & $\begin{array}{l}\text { Percentage local } \\
\text { control }\end{array}$ \\
\hline \multicolumn{6}{|l|}{ BID studies } \\
\hline Penn [7] & 32 & 45 Concurrent & 3 weeks & $48^{*}$ & $84^{\circ}$ \\
\hline ECOG-1 [8] & 41 & 45 Concurrent & 3 weeks & 36 & - \\
\hline NCI-US [9] & 35 & 45 Concurrent & 3 weeks & 65 & - \\
\hline Mayo [10] & 36 & 48 Split course & 6 weeks & 40 & 97 \\
\hline ECOG-II [1I] & 41 & 45 Alternating & 8 weeks & 40 & - \\
\hline \multicolumn{6}{|l|}{ QD studies } \\
\hline SWOG [12] & 154 & 45 Concurrent & 5 weeks & 42 & - \\
\hline Memorial [13] & 35 & 45 Sequential & $4-5$ weeks & 46 & 74 \\
\hline
\end{tabular}

All 4 variant histologies failed locally, only one pure small cell failed locally. BID=twice daily; $\mathrm{QD}=$ once daily; $E C O G=$ Eastern Cooperative Oncology Group; SWOG=Southwest Cooperative Oncology Group. 
TABLE 4

Relative scales to compare 45 Gy delivered in varied fraction sizes and times

\begin{tabular}{lllllr}
\hline Institution & Fraction $(\boldsymbol{n})$ & $\begin{array}{l}\text { Fraction size } \\
(\mathbf{G y})\end{array}$ & Time (days) & RET & NRET \\
\hline Memorial & 18 & 2.5 & 30 & 1546 & 1029 \\
SWOG & 25 & 1.8 & 33 & 1415 & 885 \\
Penn, ECOG-I & 30 & $1.5^{*}$ & 19 & 1438 & 844 \\
ECOG-II & 30 & $1.5^{*}$ & 54 & 1282 & 793 \\
\hline
\end{tabular}

Twice daily.

follow-up and patient numbers to better evaluate the merit or risks of altered fraction schemes.

Table 4 displays 4 ways to deliver 45 Gy to the chest, as delivered in 4 reported trials. The Nominal Standard Dose methods have been used to compare relative effects, the RET provides a relative scale for acute effects, and the NRET provides a relative scale for late effects. These scales may have flaws making their absolute values questionable, but they provide a reasonable mathematic model offering relative values for comparison of different fractionation on time schedules. Most believed the $45 \mathrm{~Gy}$ given in b.i.d. fashion was much more acutely toxic than the same dose administered once each day in 18-Gy fractions the two schemes used in the Intergroup Trial in the United States. The calculations predict very little difference between the two schedules, at least due to the radiotherapy factors (see SWOG vs. Penn or ECOG-I, Table 4). Differences may also be influenced by the type of and timing with chemotherapy, as well as patient and tumor factors. Only trials will determine if "breaks", time protraction of therapy, provide safer therapy or result in better, worse or similar tumor controls.

Prophylactic cranial irradiation remains a contentious issue. If it is to be used, the relative effect of fractionation may be important. Even trials that have applied doses between 2000 and 3000 rad in one to three weeks report brain failures of approximately $20 \%$. Doses using larger dose per fraction that are integrated with chemotherapy in ways that allow for an adequate blood/brain barrier protection have resulted in late imaging abnormalities and late neurologic toxicities [14-16]. There is relatively little information of using hyperfractionated schemes in prophylactic cranial irradiation. Since hyperfractionation is the best strategy to avoid unfavorable late effects, this may be a useful strategy. However, it would tax the patients to travel more frequently. Three-gray or greater fractions were probably unwise, even when delayed to completion of systemic therapy.

Perturbations in fractionation, overall time of therapy and integration of chemotherapy with local modality affords a rich vein to pursue in clinical research. There are rationales to increase effectiveness and decrease toxicity. Both are worthy of further investigation.

\section{References}

1 Bleehen NM, Bunn PA, Cox JD. Role of radiation therapy in small cell anaplastic carcinoma of the lung. Cancer Treat Rep 1983;67:11. 
2 Choi NC, Carey RC. Locoregional failure rate in relation with radiation dose in combined modality approach of multi-agent chemotherapy and radiotherapy for limited small cell lung carcinoma. Int J Radiat Oncol Biol Phys 1987;13(suppl): 188.

3 Papac RJ, Son Y, Biers R et al. Improved local control of thoracic disease in small cell lung cancer with higher dose thoracic irradiation and cyclic chemotherapy. Int J Radiat Oncol Biol Phys 1987;13:993.

4 Looney WB, Hopkins HA. Alternation of chemotherapy and radiotherapy in cancer management. III. Results in experimental solid tumor systems and their relationship to clinical studies. Cancer Treat Rep 1986;70:141162.

5 Arriagada R, LeChevalier T, Ruffie P et al. Alternating radiotherapy and chemotherapy in 173 consecutive patients with limited small cell lung carcinoma. Int J Radiat Oncol Biol Phys 1990;19:1135-1138.

6 Yaes RJ, Patel P, Maruyama Y. On using the linear-quadratic model in daily clinical practice. Int J Radiat Oncol Biol Phys 1991;20:1353-1358.

7 Muggia F, Krezoski SK, Hansen H. All kinetic studies in patients with small cell carcinoma of the lung. Cancer 1974;34:1683-1690.

8 Turrisi AT, Glover DJ. Thoracic radiotherapy variables: influence on local control in small cell lung cancer limited disease. Int J Radiat Oncol Biol Phys 1990;19:1473-1479.

9 Turrisi AT, Wagner H, Glover B et al. The Eastern Cooperative Oncology Group: limited small cell lung cancer (LSCLC): concurrent BID thoracic radiotherapy (TR) with platinum-etoposide (PE): an ECOG study (abstract). Proc ASCO 1990;9:230.

10 Johnson BE, Salem C, Nesbitt J et al. Limited (LTD) stage small cell lung cancer (SCLC) treated with concurrent bid chest radiotherapy (RT) and etoposide cisplatin (VP/PT) followed by chemotherapy (CT) selected by in vitro drug sensitivity testing (DST) (abstract). Lung Cancer 1991;7(Suppl):152.

11 Frytak S, Shaw E, Eagan $R$ et al. Accelerated hyperfractionated split-course thoracic radiotherapy (AHSCTRT) and infusion cisplatin based chemotherapy (CT) for small cell lung cancer (abstract). Lung Cancer 1991;7(suppl): 157 .

12 Johnson DH, Turrisi AT, Chang AY, Wagner H, Blum R. The Eastern Cooperative Oncology Group. Alternating chemotherapy (CT) \& thoracic radiotherapy (TRT) in limited small cell lung cancer (abstract). Lung Cancer 1991;7(suppl): 155 .

13 McCracken JD, Janaki LM, Crowley JJ et al. Concurrent chemotherapy/radiotherapy for limited small-cell lung carcinoma: a Southwest Oncology Group study. J Clin Oncol 1990;8:892.

14 Shank B, Scher H, Hilaris RS et al. Increased survival with high-dose multifield and intensive chemotherapy in limited small cell carcinoma of the lung. Cancer 1985;56:2771.

15 Turrisi AT. Brain irradiation and systemic chemotherapy for small-cell lung cancer: dangerous liaisons? J Clin Oncol 1990;8:196-199.

16 Fleck JF, Einhorn LH, Lauer RC, Schulz SM, Miller ME. Is prophylactic cranial irradiation indicated in small cell lung cancer? J Clin Oncol 1990;8:209-214.

17 Lishner M, Feld R, Payne DG et al. Late complications after prophylactic cranial irradiation in patients with small-cell lung cancer: the Toronto experience. J Clin Oncol 1990;8:215-221. 